\title{
Modificaciones de diseño protésico para mejorar estética en prótesis parcial removible metálica
}

\section{Modifications in removable partial dentures design looking for better aesthetic results}

\author{
Fernández $\mathrm{E}^{*}$, Monardes $\mathrm{H}^{* *}$, Mardones $\mathrm{F}^{* * *}$, Acosta $\mathrm{H}^{* * * *}$, Segovia JL****, \\ Román $\mathrm{J}^{* * * *}$, Bernal $\mathrm{G}^{* * * * *}$
}

\section{RESUMEN}

Pese al innegable incremento en la utilización de implantes óseo integrados en el tratamiento de la extensa problemática de la desdentación parcial, la confección de prótesis removibles metálicas continua siendo a escala mundial, el principal recurso terapéutico vigente. Sin embargo, desde el punto de vista del paciente, la recuperación de la función estética es su principal falencia. Proponemos entonces, extender la utilización del principio de retención friccional inherente a la prótesis fija, mediante la preparación de" facetas retentivas friccionales" en ciertos dientes pilares, generando de este modo artificios protésicos removibles con suficiente retención, sin exhibir elementos metálicos en los sectores anteriores de la cavidad bucal. Se exponen cinco casos clínicos que respaldan la validez nuestra propuesta tras una correcta indicación.

Palabras clave: Prótesis removible, retención, estética, facetas retentivas.

\section{SUMMARY}

Despite the fact that nowadays the use of osseointegrated implants is increasing worldwide nevertheless removable partial dentures with cast metal frameworks is still the most indicated treatment for cases of partial edentulism. However, patients remain reluctant regarding the aesthetic impairment brought by some of the removable partial dentures' components. Therefore we propose to apply to removable partial dentures some of the frictional retention's principles used in fixed prostheses, preparing instead of conventional guiding planes in some abutment teeth, a more wide and extensive platform what we so-called "frictional retentive facets". Accordingly we can afford to provide enough retention to our removable devices without exposing unaesthetic components such as vestibular retentive arms. Five clinical cases will be shown in order to sustain our proposal keeping in mind the need of respecting the right indication.

* Licenciado en Odontología. Universidad de Chile. Especialidad en Prótesis Dental, Université Catholique de Louvain. Docente del V año de Clínicas Integradas y del Postgrado en Rehabilitación Oral de la Facultad de Odontología. Universidad San Sebastián. Santiago de Chile.

** Cirujano-dentista, Universidad de Concepción. Magíster en Ciencias Biomédicas, Universidad de Talca. Docente del V año de Clínicas Integradas y Director del Postgrado de Endodoncia de la Facultad de Odontología, Universidad San Sebastián, Santiago de Chile.

*** Cirujano-dentista, Universidad de Chile, alumno del Postgrado en Rehabilitación Oral, Versión 20102011. Universidad San Sebastián. Santiago de Chile.

**** Alumnos de V año 2011, Clínicas Integradas, Facultad de Odontología. Universidad San Sebastián. Santiago de Chile. 
Key words: Removable prostheses, retention, aesthetic, retentive facets.

Fecha de recepción: 23 de marzo de 2012.

Aceptado para publicación: 25 de mayo de 2012.

Fernández E, Monardes H, Mardones F, Acosta H, Segovia JL, Román J, Bernal G. Modificaciones de diseño protésico para mejorar estética en prótesis parcial removible metálica. Av. Odontoestomatol 2013; 29 (4): 175-183.

\section{INTRODUCCIÓN}

El término plano guía se refiere a dos o más superficies verticales paralelas en los dientes pilares diseñadas para dirigir la prótesis durante su inserción y remoción. Ésta es su principal función. Las superficies de los planos guía deben quedar tan paralelas al eje mayor del diente pilar como sea posible (1).

Los planos guía así elaborados para determinar un eje único de inserción y remoción de la prótesis esquelética o metálica proveen a ésta de cierta retención por fricción, generada por el íntimo o estrecho contacto del elemento metálico de la base en las superficies dentarias de los pilares para aquellos fines paralelizados (planos guía).

Este tipo de "retención friccional" (F.R.), que buscamos y utilizamos de manera fundamental en prótesis fija, (2) adquiere en prótesis removible un papel secundario en comparación con la retención entregada por la energía cinética que genera la flexión de un brazo retentivo al flanquear el ecuador protésico.

Sin embargo creemos que el papel secundario de la R.F. obtenida en los planos guía, pudiese adquirir mayor preponderancia en la retención del aparato protésico removible, si introdujésemos algunas modificaciones que remiten tanto a la extensión en la preparación, como al maxilar y al tipo de desdentación que presenta el caso clínico a tratar (clasificación de Kennedy) De este modo, podríamos lograr una gran ventaja estética en la prótesis puesto que la base metálica así confeccionada, no emplazaría brazos retentivos visibles en vestibular, en los sectores anteriores de la boca.
Al extender al máximo el desgaste que efectuamos en la cara cercana al vano, en sentido axial, cérvicooclusal y vestíbulo palatino o lingual, obtendremos una superficie para fricción mucho mayor que la de un pequeño plano guía, es decir, se constituye en una "Faceta Retentiva Friccional" (F.R.F.).

\section{MATERIAL Y MÉTODOS}

Analizaremos cinco casos clínicos en los cuales, con mayor y menor éxito (léase fracaso), intentamos aplicar estos conceptos para beneficio estético de los pacientes, sin atentar contra el esencial requisito de salvaguardar la Retención del aparato protésico, capital para recuperar funciones. Los casos fueron tratados durante 2011 por alumnos del V año de Clínicas Integradas de la USS, sede Santiago, y un colega, alumno del postgrado de Rehabilitación Oral de la misma casa de estudios.

Los procedimientos clínicos y la secuencia de diagnóstico, análisis y ejecución de estas prótesis removibles metálicas, no difieren de los tradicionales a excepción de los que dicen relación con la llamada preparación bioestática o preparación de dientes pilares para fines protésicos, que detallaremos a continuación.

\section{DIFERENCIAS EN LAS DIMENSIONES DE LAS F.R.F. COMPARADAS CON PLANOS GUIIA}

Según McCracken (1), la superficie de los planos guía debe ser parecida a un objeto cilíndrico, lisa, continua y con ángulos redondeados. Como norma ge- 
neral, las superficies guía deben abarcar la mitad de la anchura que existe entre las puntas de las cúspides vestibulares y linguales adyacentes, o un tercio de la distancia vestíbulo lingual del diente. Se deben extender verticalmente unos dos tercios de la longitud del esmalte de la corona desde la cresta marginal, en dirección cervical.

Sin embargo, las F.R.F. presentan las siguientes peculiaridades en su preparación (Figs. 1 a 4):

1. En un sentido axial respecto del eje mayor del diente, el desgaste deberá eliminar prácticamente toda la convexidad "natural" del diente intervenido, desgaste no menor de 0,5 mm. Caninos superiores e inferiores, premolares superiores,

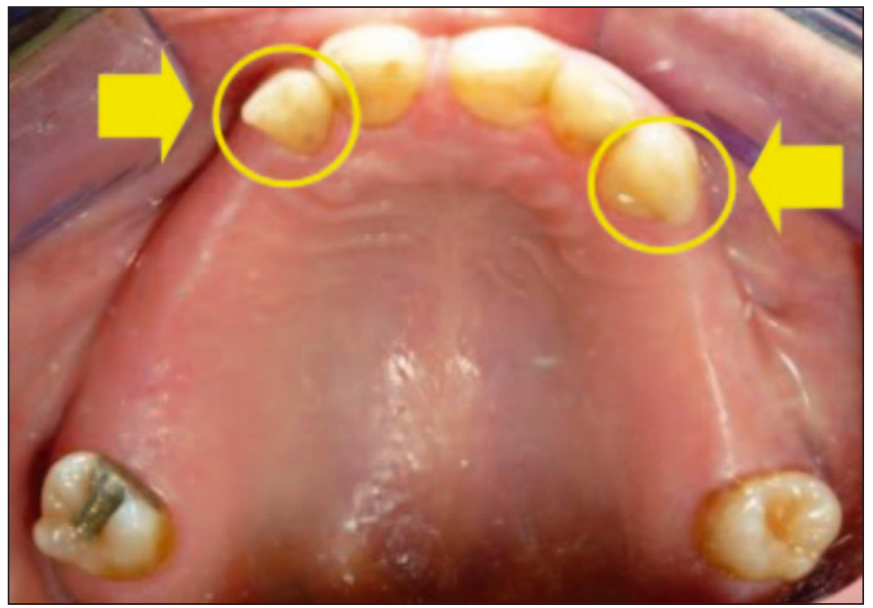

Figura 1

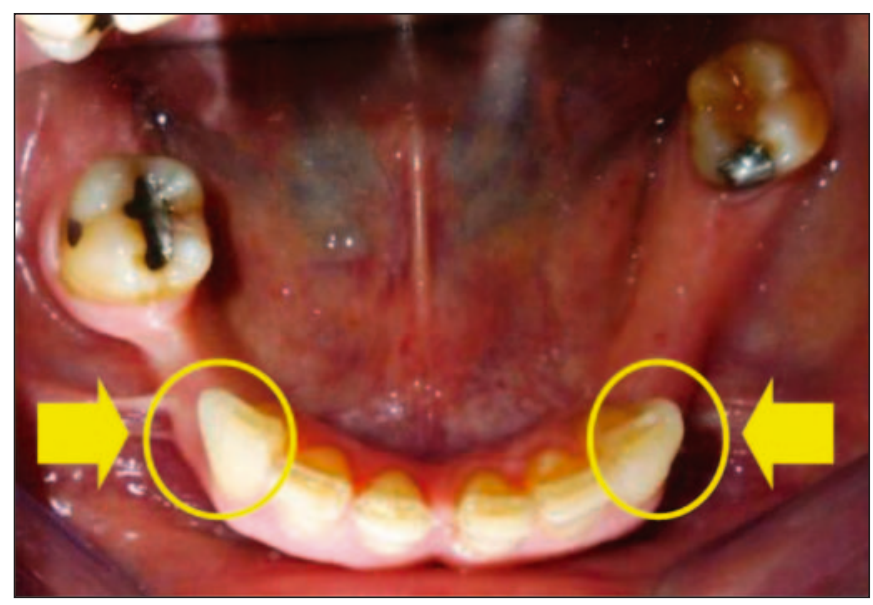

Figura 2 premolares inferiores, laterales superiores y laterales inferiores, en este respectivo orden, toleran sin mayores efectos inflamatorios pulpares estas preparaciones pudiendo al finalizar el procedimiento, de presentarse tales efectos secundarios, aplicar flúor barniz (22.600 p.p.m.)

2. El desgaste de la F.R.F. en el sentido cérvico oclusal/inciso cervical debe ocupar la máxima dimensión posible desde la cresta marginal, pero manteniendo hacia cervical una distancia mínima de $1 \mathrm{~mm}$ respecto de la encía marginal, similar en este punto a lo indicado para un complejo retentivo (C.R.) (3) del tipo R.P.I. (4) En prótesis fija, se recomienda una dimensión cérvico oclusal/inciso cervical no menor de $3 \mathrm{~mm}$ para incisivos, caninos y premolares (2).

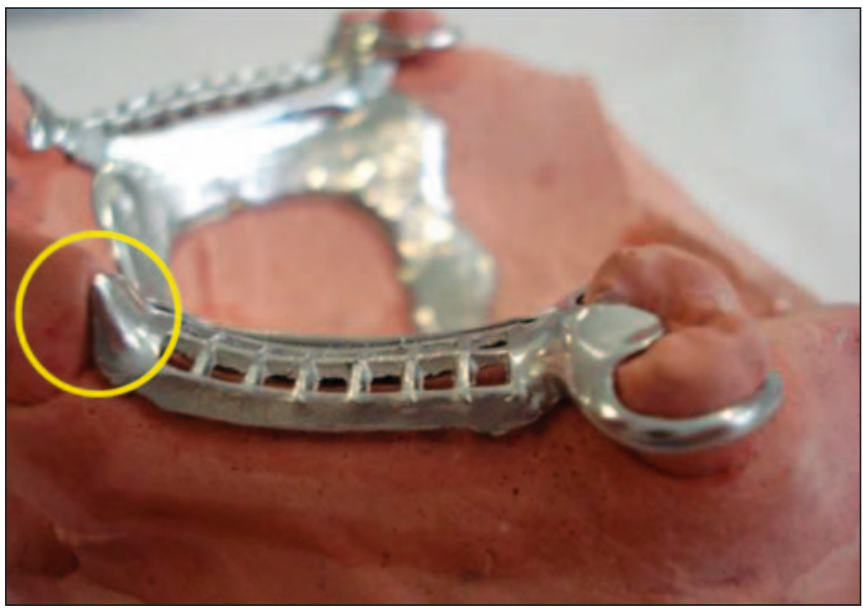

Figura 3

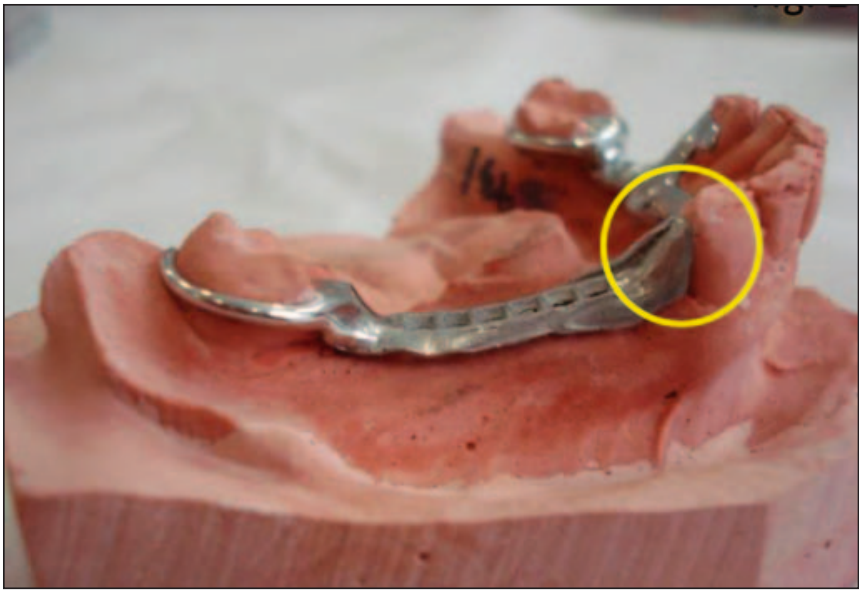

Figura 4 
3. En sentido vestíbulo palatino o lingual, (o como lo denomina Goodacre, faciolingual), la F.R.F. debe abarcar la máxima extensión posible en la cara sometida a desgaste, teniendo la prudencia de no "intervenir" parte de la cara vestibular, puesto que así disminuiría la ventaja estética que buscamos al no tener elemento metálico a la vista en el diente pilar.

4. Al preparar las F.R.F., al igual que con los planos guía, hay que tener especial cuidado en no crear líneas-ángulo, vestíbulo linguales o palatinas, ya que, de entre las muchas razones aducidas, los ángulos lineales agudos crean una sobreconcentración de stress, así como dificultan ciertos procedimientos de laboratorio (2).

\section{RESULTADOS}

\section{Caso clínico 1}

Paciente género femenino, $4^{\mathrm{a}}$ década, clase III de Kennedy, maxilar y mandibular, facetas retentivas friccionales (F.R.F.) en 1.3, 2.2 y 3.3 y 4.3 (Figs. 5 a 10).

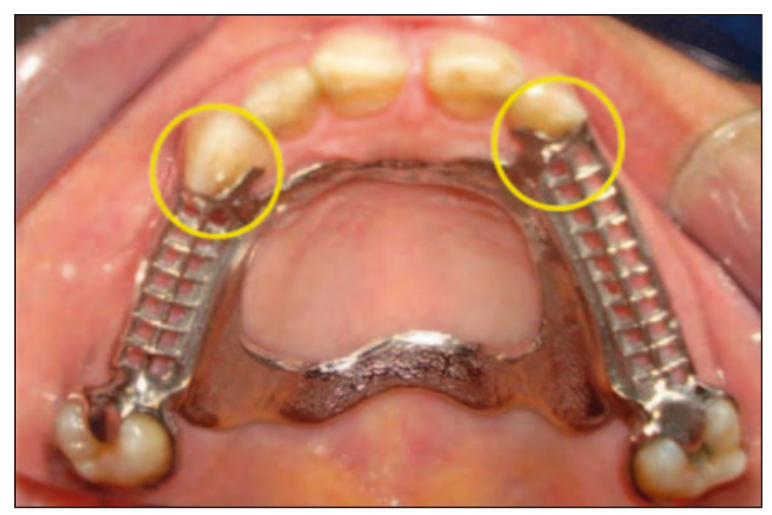

Fig. 5

Fig. 6
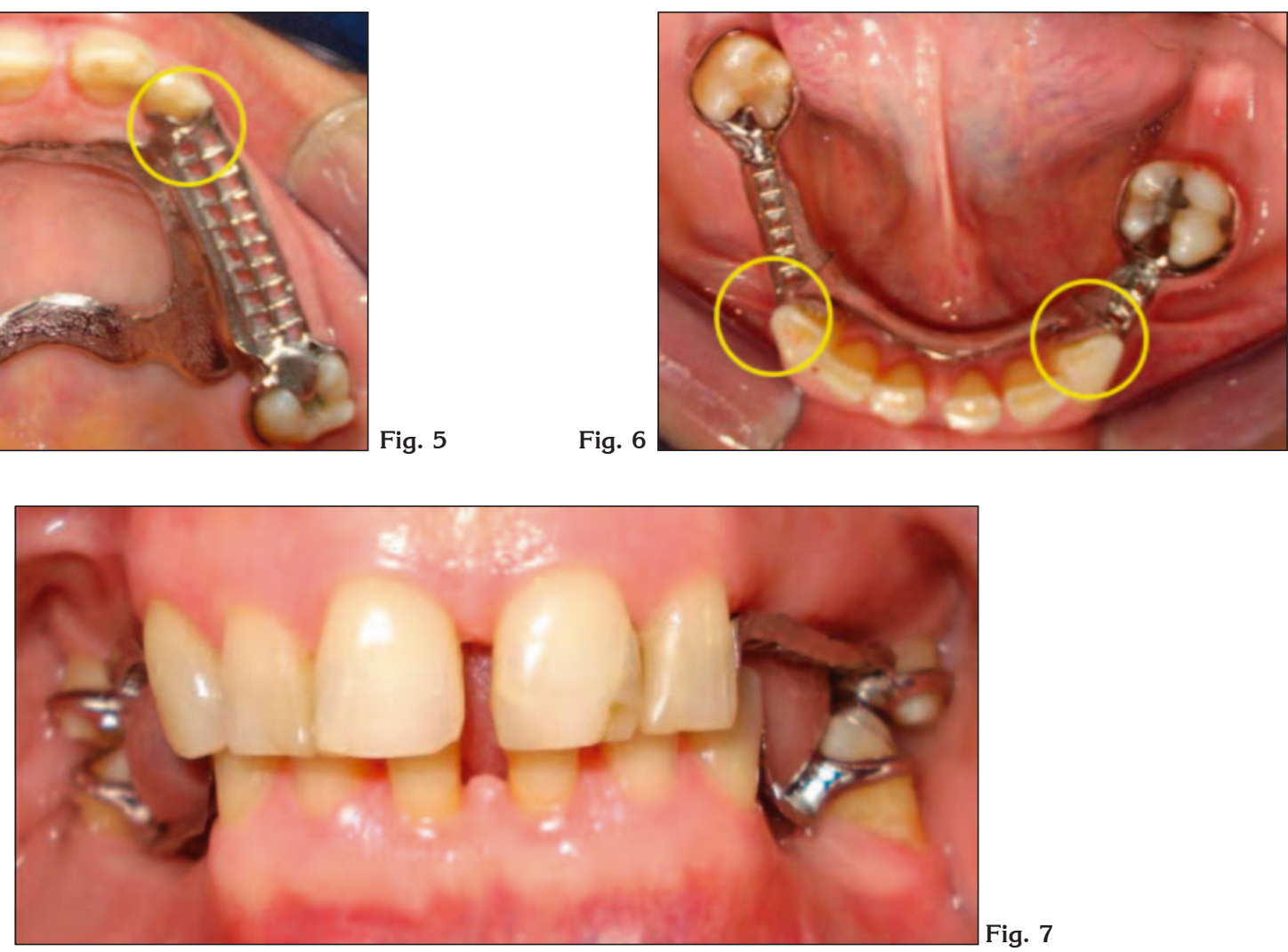

Fig. 7

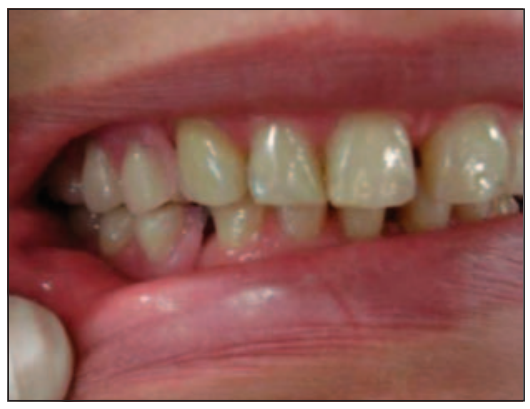

Fig. 8

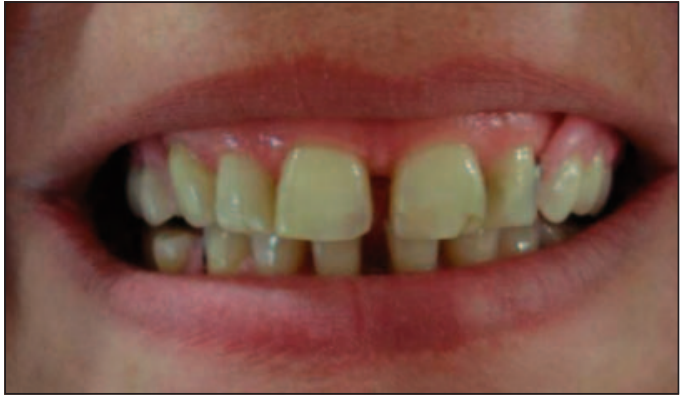

Fig. 9

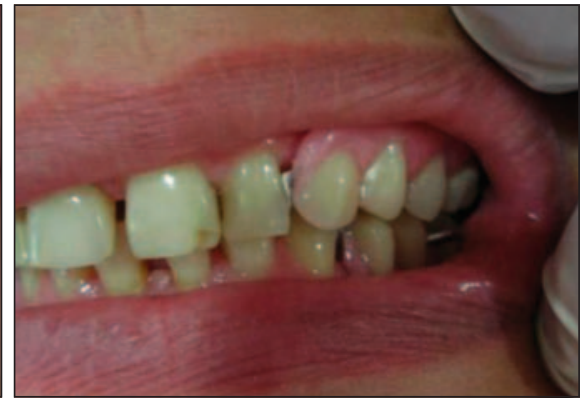

Fig. 10 


\section{Caso clínico 2}

Paciente género masculino, $4^{\mathrm{a}}$ década, Clase II de Kennedy maxilar, subdivisión 2, F.R.F. en 1.3 y 2.3/Clase I de Kennedy mandibular, subdivisión 1 , complejos retentivos R.P.I. y Akers (Figs. 11 a 14).

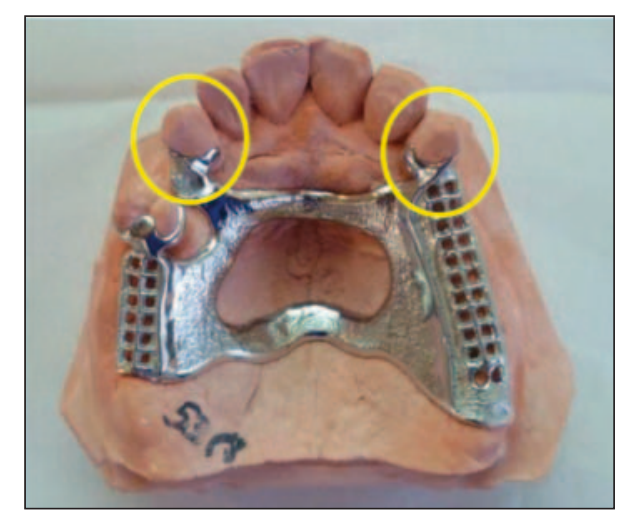

Fig. 11

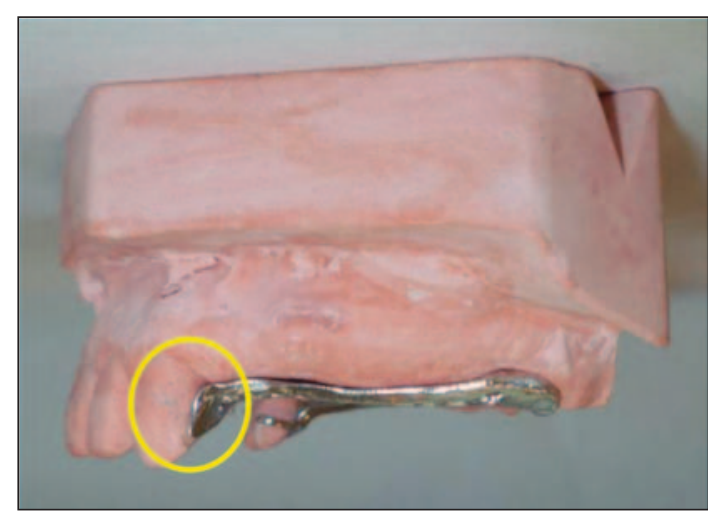

Fig. 12

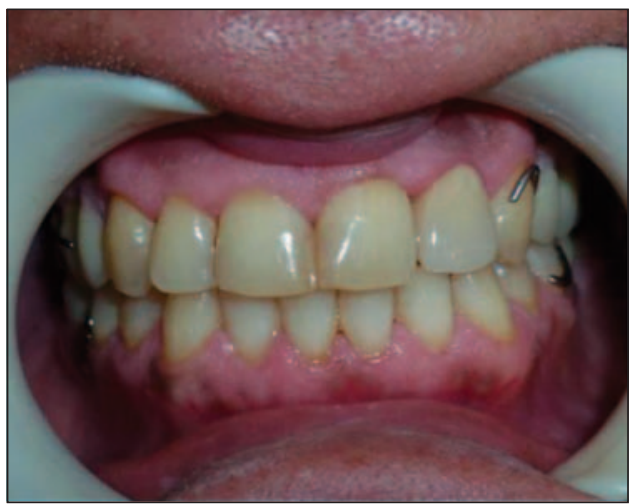

Fig. 13

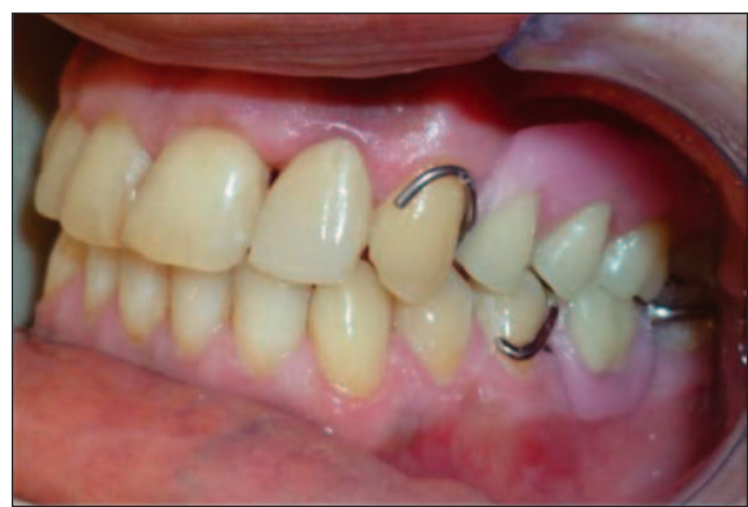

Fig. 14

\section{Caso clínico 3}

Paciente género femenino, $7^{\text {a }}$ década, clase III de Kennedy maxilar, subdivisión 1, F.R.F. en 1.3, 2.3 1.7 y 2.7 con retenedores intrarradiculares en $1.5 \mathrm{y}$ 2.5/clase I de Kennedy mandibular (Figs. 15 a 22).

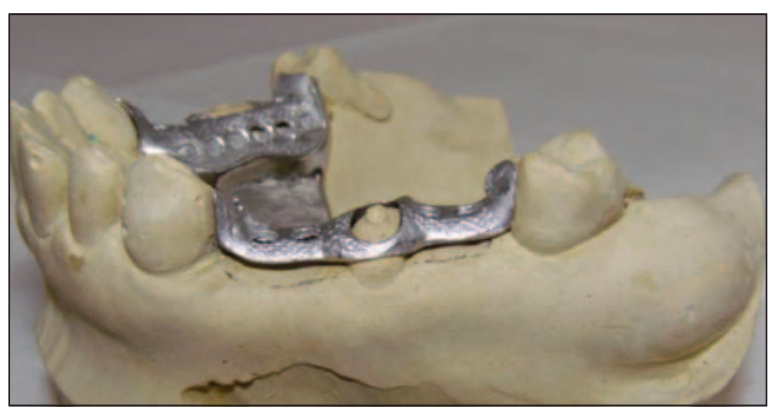

Fig. 15

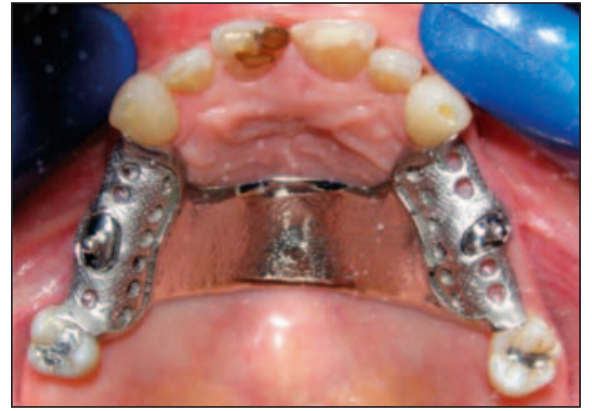

Fig. 18

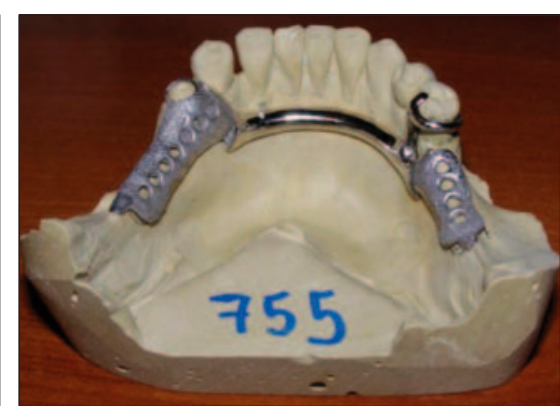

Fig. 16

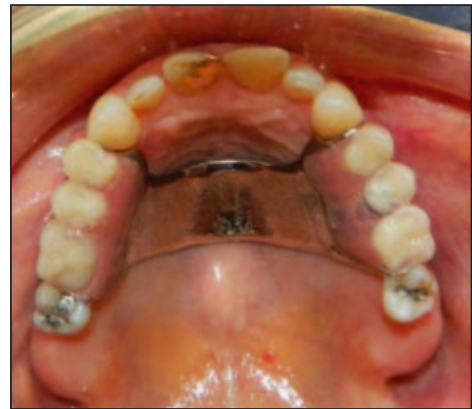

Fig. 19
Fig. 17

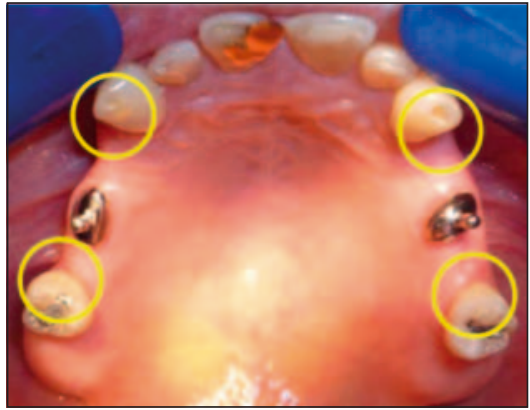




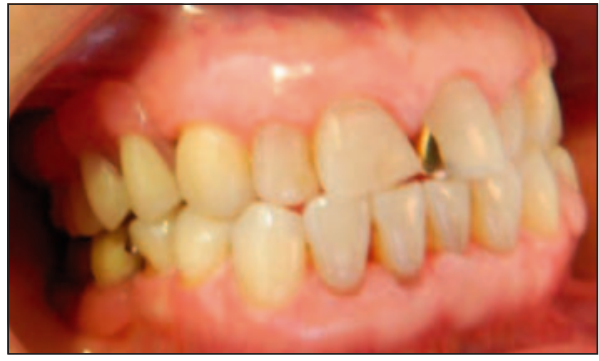

Fig. 20

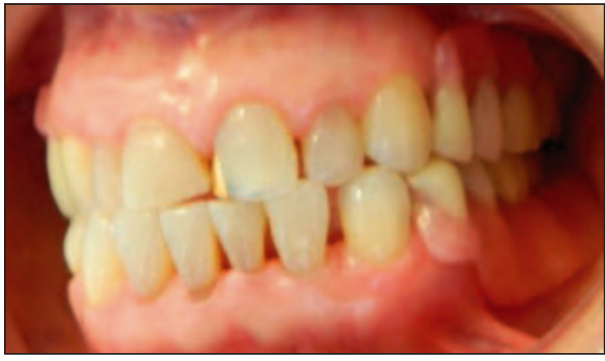

Fig. 21

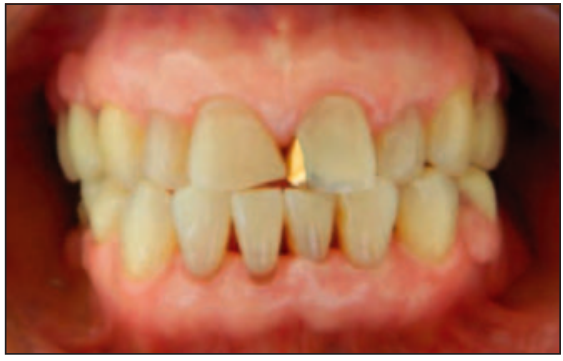

Fig. 22

\section{Caso clínico 4}

Paciente género femenino, $3^{a}$ década, clase III de Kennedy, subdivisión 2, maxilar. F.R.F. en 1.1, 1.3 y 2.3/maxilar inferior, clase III de Kennedy, subdivisión 1 , rehabilitada mediante prótesis fija plural, ceramo metal, convencional (Figs. 23 a 30).

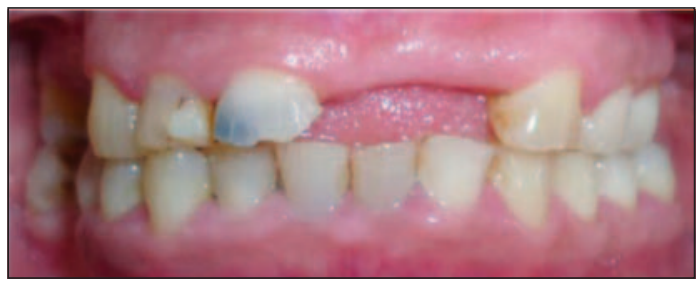

Fig. 23

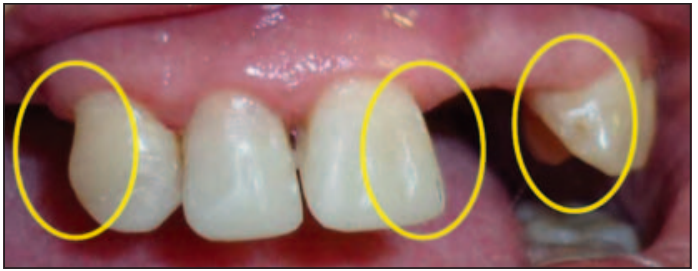

Fig. 24

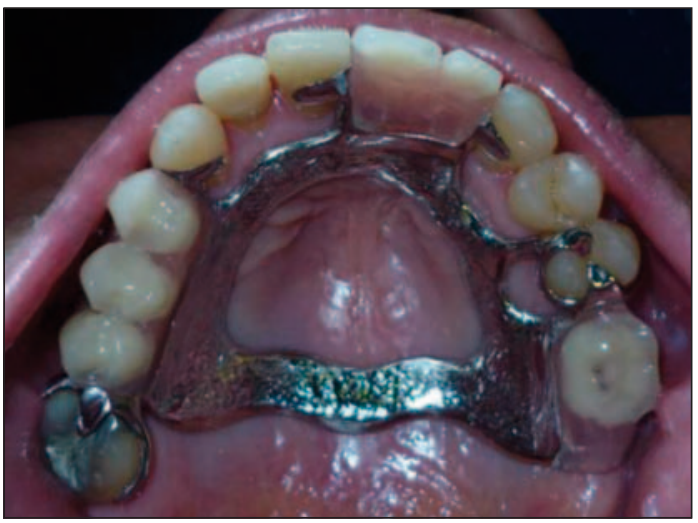

Fig. 25
Fig. 26

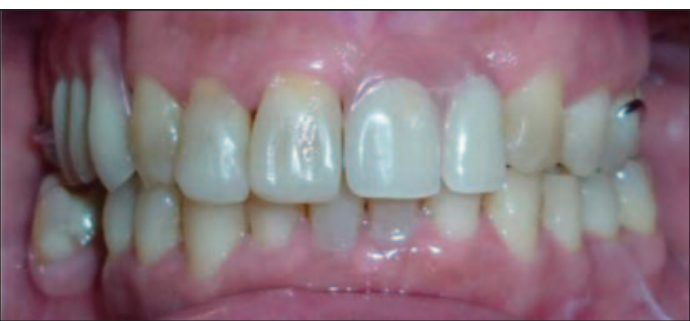

Fig. 27

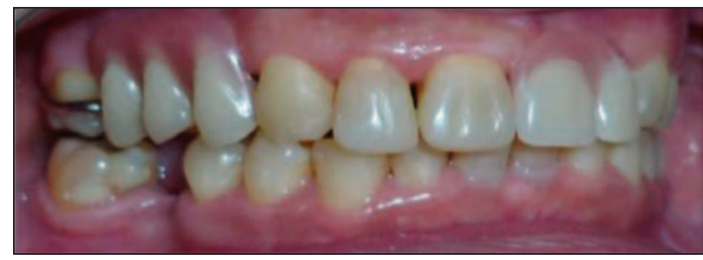

Fig. 28

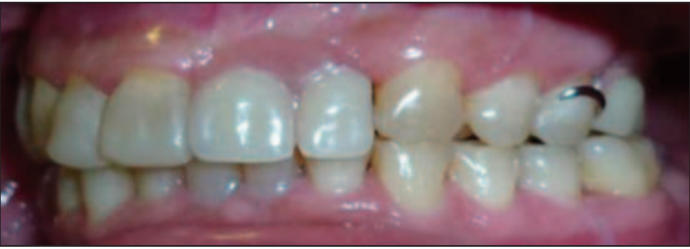

Fig. 29

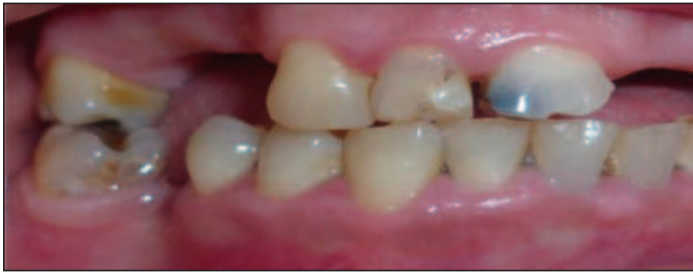

Fig. 30

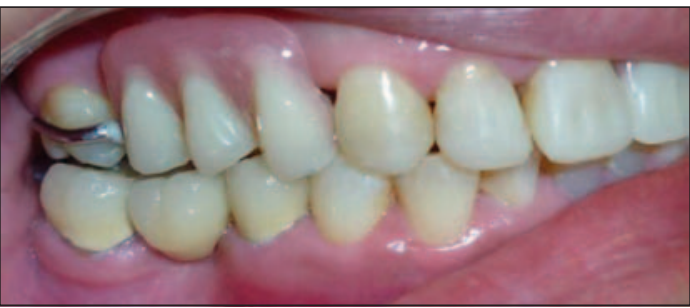


Fernández E, Monardes H, Mardones F, Acosta H, Segovia JL, Román J, Bernal G..

\section{Caso clínico 5}

Paciente género femenino, $3^{a}$ década, clase III de Kennedy, subdivisión 2, maxilar, F.R.F. en 1.3 y 2.3/maxilar inferior, clase I de Kennedy (Figs. 31 a 38).

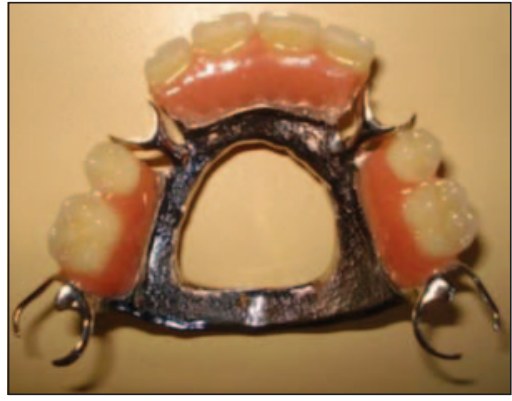

Fig. 31

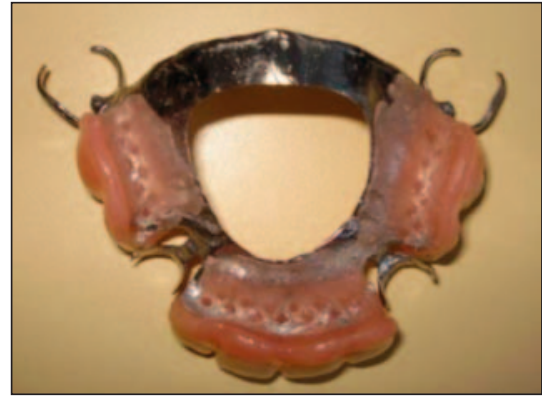

Fig. 32

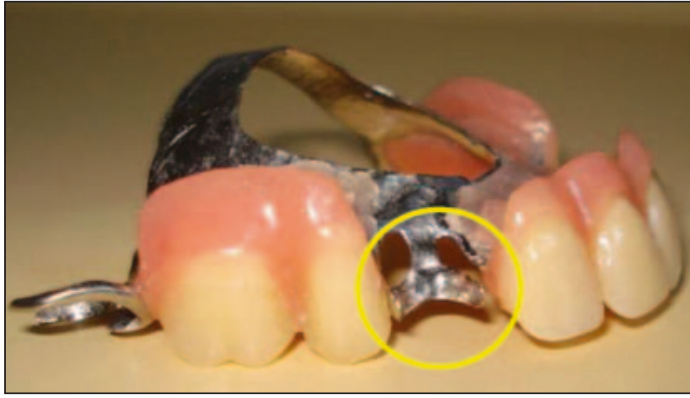

Fig. 33

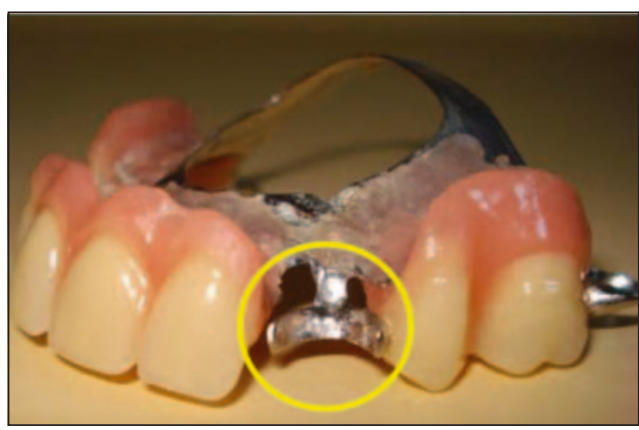

Fig. 34

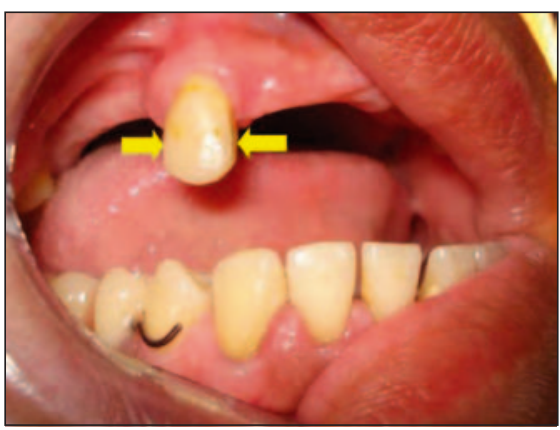

Fig. 35

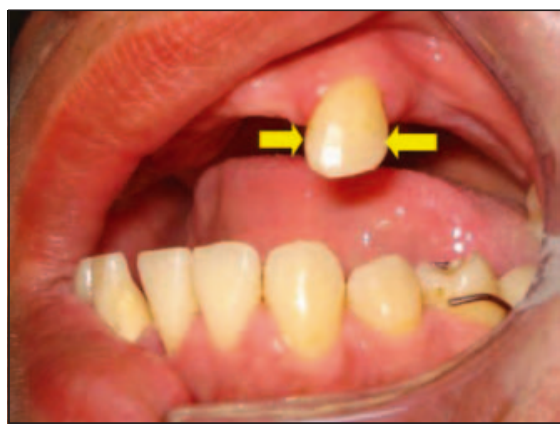

Fig. 36

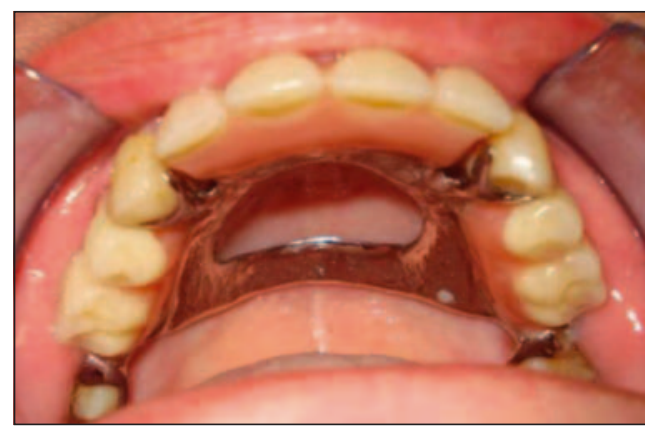

Fig. 37

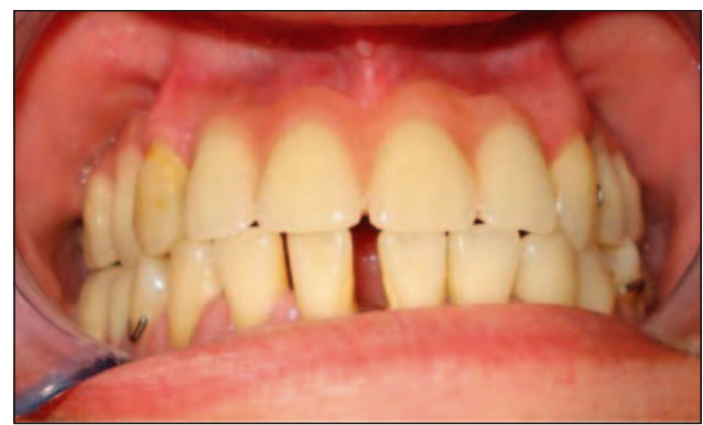

Fig. 38

\section{DISCUSIÓN}

Hemos afirmado que para obtener las ventajas de las F.R.F., se debe acotar la indicación en ambos maxilares, a clases III de Kennedy-Applegate, sean éstas puras o subdivisiones.
En este sentido, el caso clínico 1 corresponde exactamente a lo anteriormente explicitado pese a que en uno de los pilares maxilares intervenidos, 2.2, al ser un incisivo lateral, el provecho estético es menor. En maxilar inferior, se invirtió la búsqueda de retención en los molares inferiores, desde vestibular hacia 
lingual, puesto que la inclinación natural de dichos dientes así lo indicó en concordancia con el eje de inserción seleccionado. En estos pilares "posteriores" sí colocamos complejos retentivos convencionales, como son los de Akers.

Sin embargo, en los pilares "anteriores", del complejo retentivo, sólo emplazamos un apoyo oclusal o cingular para la transmisión de cargas masticatorias (soporte), y un conector menor, obviando los brazos activos y de contención reemplazados por las ya explicitadas F.R.F.

Como argumento que ratifica lo expuesto en el encabezado de este capítulo, el caso clínico 2 nos demuestra fehacientemente que no podemos sobre solicitar el recurso de las F.R.F. en clases I y II de Kennedy, en especial en el maxilar superior. En este caso en particular, la longitud del vano izquierdo, sin pilar posterior, en conjunto con la fuerza de gravedad, no generó mínimos niveles de retención compatibles con la recuperación de funciones. Se debió suplementar la retención mediante un brazo retentivo labrado de $0,9 \mathrm{~mm}$ en el diente 2.3 .

El caso clínico 3, en el maxilar superior, constituye una singularidad en la indicación de este recurso, pues nos permitió recurrir a las F.R.F. complementando la función de retenedores intrarradiculares colados "tipo Sandri", sin emplazar complejos retentivos en ninguno de los pilares, logrando así un resultado estético óptimo.

De este modo las F.R.F., en caninos y molares que limitan los vanos, aportaron guía de inserción y estabilización de la prótesis, delegando mayoritariamente el soporte en el hueso alveolar y la retención principal, en los dispositivos intrarradiculares. Así, evitamos la generación de otros fulcros que no fuere el que se establece en relación a estos pernos situados simétricamente en dientes 1.5 y 2.5. En este caso, las F.R.F. ejercerán de modo marginal como elementos de retención, puesto que ésta es asumida esencialmente por los retenedores intrarradiculares.

En el maxilar inferior, rehabilitación "convencional" mediante "attache intrarradicular" tipo "Sandri" en diente 3.4 y complejo retentivo Nally-Martinet en diente 4.5 .
En esta misma perspectiva de complementariedad de las F.R.F., el caso clínico 4 nos demuestra su utilidad al indicarla en el maxilar superior, como alternativa de recuperación estética aceptable en sector anterior mediante una prótesis parcial removible, lo que habitualmente resulta en un desafío no logrado mediante estos artificios, fundamentalmente, debido a la intrínseca dificultad de "compatibilizar" la apariencia estética de piezas naturales con la de los dientes artificiales, así como por la "exposición" estéticamente desafortunada de brazos retentivos.

Cabe destacar que la confección de F.R.F. no es privativa de dientes naturales como lo demuestra la figura 24 , dientes 1.3 y 2.3 indemnes y diente 1.1 , restaurado en conjunto con 1.2 , mediante carillas estéticas directas con resina compuesta.

Finalmente, el caso clínico 5 presenta una alternativa de tratamiento para pacientes jóvenes que habiendo sufrido múltiples pérdidas de dientes en el maxilar superior, aquellos remanentes lo hacen en una distribución en la arcada superior que posibilita rehabilitar mediante prótesis removible recuperando función y estética. En los caninos 1.3 y 2.3, F.R.F. en caras mesial y distal y confección de C.R. del tipo "pinzas mesiodistales de Roach" (Figs. 33 y 34).

Además, la omnipresente y desfavorable variable económica, determinó canalizar recursos para acometer en una segunda fase, tras el cierre de diastemas mediante ortodoncia, la rehabilitación del maxilar inferior mediante prótesis implanto retenidas, puesto que sabemos que en una desdentación de extremo libre bilateral, muy pocas veces se puede recuperar satisfactoriamente estética y función, mediante dispositivos protésicos removibles.

\section{CONCLUSIONES}

Creemos que, en la odontología actual, la prótesis parcial removible esquelética o metálica continúa siendo un recurso terapéutico plenamente vigente para una vasta población del planeta. Por tanto, debemos perseverar en la búsqueda de mejoras y la variación del diseño protésico aportado por las F.R.F., 
para casos clase III de Kennedy y subdivisiones, constituye una contribución.

Esta pertinente indicación con la presencia ojalá simétrica de piezas posteriores, nos permite emplazar en ellas complejos retentivos convencionales cuyos brazos retentivos de mayor grosor aportarán y "compensarán" la disminución de cierta retención en el sector anterior. El ganancial es estético, al no tener que utilizar brazos activos en vestibular de piezas anteriores o visibles.

No debemos extrapolar la utilización de F.R.F. a casos de desdentación con extremos libres, Kennedy I y II, toda vez que su aporte a la retención total del aparato protésico se demostró insuficiente.

Los casos de clase IV de Kennedy pueden ser abordados con buenos resultados estéticos y funcionales, mediante la utilización de ejes de inserción rotacionales ampliamente difundidos en nuestra especialidad, desde la década de los setenta del siglo recién pasado.

\section{BIBLIOGRAFÍA}

1. Carr AB, McGivney GP, Brown DT. Principios de Diseño de Prótesis Parciales Removibles. En:
McCracken Prótesis Parcial Removible. Madrid: Elsevier España S.A, 2006:154-5.

2. Goodacre ChJ, Campagni WV, Aquilino SA. Preparaciones dentarias para coronas completas: una forma de arte basada en principios científicos, J Prosthet Dent 2001;85:363-76.

3. Grimonster J, Fernández E, Vanzeveren Ch. Prothèse amovible partielle: les grands príncipes régissant la conception et la réalisation d'une PAP. -Editions Techniques- Encycl. Méd. Chir. (ParisFrance), Odontologie, $23310 \mathrm{C}^{10}, 1991,26$ p.

4. Fernández E, Grimonster J. Retención y biomecánica de los complejos retentivos III parte: la Escuela de Kratochvil y complejos retentivos actuales, Av Odontoestomatol 1989;5:180-7.

\section{CORRESPONDENCIA}

Enrique Fernández $M$.

Facultad de Odontología

Universidad San Sebastián

Av. Cumming, 40

Santiago de Chile

E-mail: efernandez@santotomas.cl 